\title{
Approaching the Mechanistic Insights Towards the Genesis of Intracellular Calcium Transient Alternans - a Simulation Study
}

\author{
H Zhang, T Tao, SC O'Neill
}

\author{
University of Manchester, Manchester, UK
}

\begin{abstract}
Mechanical contraction alternans of the heart is associated with fatal cardiac death. It is manifested by $T$ wave alternans in the ECG, and is thought to be possibly related to intracellular $\mathrm{Ca}^{2+}$ transient alternans released from the sarcoplasmic reticulum (SR). However, it is unclear yet how beat-to-beat alternans of intracellular $\mathrm{Ca}^{2+}$ transient is produced. In this study investigated the mechanism(s) underlying the genesis of intracellular $\mathrm{Ca}^{2+}$ alternans produced at slow pacing rates by using a mathematical model of a spatially extended cardiac cell with a cluster of coupled ryanodine receptor $(R y R)$ elements. It was shown that the intracellular $\mathrm{Ca}^{2+}$ alternans was generated by propagating waves of $\mathrm{Ca}^{2+}$ release and sustained through alternation of $S R \mathrm{Ca}^{2+}$ content that has a stiff relationship with the $\mathrm{Ca}^{2+}$ transient. This study provides novel and fundamental insights to understand mechanisms that may underlie intracellular $\mathrm{Ca}^{2+}$ alternans without the need for refractoriness of L-type Ca or RyR channels under rapid pacing.
\end{abstract}

\section{Introduction}

Mechanical contraction alternans is commonly observed in patients with heart failure, with which the force of heart contraction alternates between strong and weak leading to sudden cardiac death [1-2]. Mechanical contraction alternans is manifested by $\mathrm{T}$-wave alternans in the ECG, and is thought to be possibly related to intracellular $\mathrm{Ca}^{2+}$ transient alternans released from the sarcoplasmic reticulum (SR), the main source of $\mathrm{Ca}^{2+}$ responsible for cardiac contraction [3-6]. However, it is unclear yet how beat-to-beat alternans of intracellular $\mathrm{Ca}^{2+}$ transient is produced.

The work presented here attempts to investigate the mechanism(s) underlying the genesis of intracellular $\mathrm{Ca}^{2+}$ alternans. We utilizes a biophysically detailed computer model for the $\mathrm{Ca}^{2+}$ release and propagation in a spatially extended cardiac ventricular myocytes to underpin detailed mechanisms responsible for the emergence of cytoplasmic $\mathrm{Ca}^{2+}$ transient alternans as observed in our previous experiments [7-8]. Our simulation data shows that alternans can arise due to propagation of $\mathrm{Ca}^{2+}$ waves and sustain through alternation of sarcoplasmic reticulum (SR) $\mathrm{Ca}^{2+}$ content that leads to a large increase in the gain of the feedback controlling SR $\mathrm{Ca}^{2+}$ content. This mechanism differs to the mechanism of refractoriness of RyR gate, by which $\mathrm{Ca}$ alternans can be produced by rapid pacing. Our study provides novel and fundamental insights to understand mechanisms underlying the genesis of intracellular $\mathrm{Ca}^{2+}$ alternans at low excitation rates.

\section{Methods}

A mathematical model of a spatially extended cardiac cell has been developed and is utilized in this study. In the model, a general cardiac ventricular cell is considered with a length of $150 \mu \mathrm{m}$, which is discretized by a spatial resolution of $6 \mu \mathrm{m}$ to form 25 coupled ryanodine receptor (RyR) elements. This model has a spatial resolution coarser than $2 \mu \mathrm{m}$ as used in our previous study [9], but is much more computationally economic and produces similar results to our previous model [9]. Each element of the model has a cluster of unitary voltage-gated L-type $\mathrm{Ca}^{2+}$ channels, a subspace under the sarcolemma, a cytoplasmic space and a cluster of sarcolemma reticulum (SR) RyR channels. For each element, mathematical equations were developed to model $\mathrm{Ca}^{2+}$ cycling. Interelement coupling is via $\mathrm{Ca}^{2+}$ diffusion from subspaces to cytoplasmic spaces and via network SR spaces. In simulations, two protocols were used to produce $\mathrm{Ca}^{2+}$ alternans, both of which used $100 \mathrm{~ms}$ depolarising pulses with a low frequency at $1 \mathrm{~Hz}$ from a holding potential of $40 \mathrm{mV}$ to activate L-type channel opening. In the first protocol, the depolarising pulse was from $-40 \mathrm{mV}$ to 0 $\mathrm{mV}$ and the $\mathrm{Ca}^{2+}$ release mechanism was partially inhibited by increasing the threshold of $\mathrm{RyR} \mathrm{Ca}{ }^{2+}$ release (mimicking the decreased sensitivity of RyR by tetracaine as suggested by a previous experimental study [7]). In the second approach L-type Ca channel openings was reduced by depolarising to only $-20 \mathrm{mV}$ and a random block of 17 out of 25 channels was applied, thus $\mathrm{Ca}^{2+}$ release was activated at only a few sites on the SR (mimicking a small depolarising pulse in a previous 
experimental study [8]). In both approaches, the relationship between the variation in the SR content and the amplitude of the intracellular $\mathrm{Ca}^{2+}$ transient was measured.

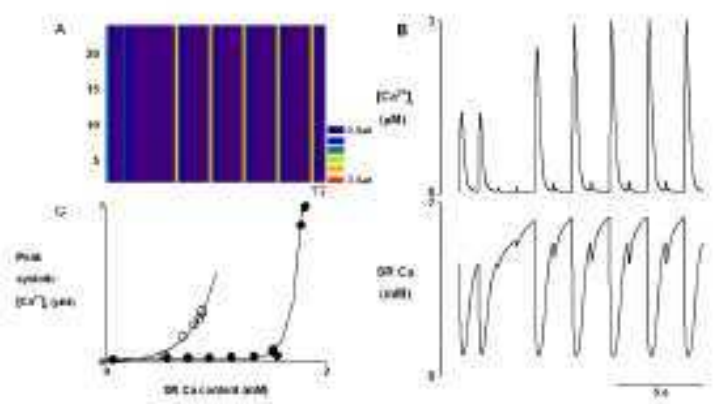

Figure 1. Model generated $\mathrm{Ca}^{2+}$ alternans by inhibiting $\mathrm{SR} \mathrm{Ca}^{2+}$ release. Ca transients were produced by a sequence of $1 \mathrm{~Hz} 100 \mathrm{~ms}$ depolarising pulses from a holding potential of $-40 \mathrm{mV}$ to $0 \mathrm{mV}$. RyR release threshold $\left(\mathrm{K}_{\mathrm{rel}}\right)$ was increased uniformly by $20 \%$ across the cell after the first 2 pulses. A) Space-time plot of colour-scaled cytoplasmic $\mathrm{Ca}^{2+}$ concentration across the cell. $\mathrm{Ca}^{2+}$ alternans was developed after a period of $\mathrm{Ca}$ release inhibition after $\mathrm{K}_{\mathrm{rel}}$ was increased. B) Time traces of cytoplasmic $\mathrm{Ca}^{2+}$ and $\mathrm{SR} \mathrm{Ca}^{2+}$ transients. C) Computed relationship between SR Ca content and systolic $\mathrm{Ca}$ in the cytoplasmic space for control (open symbols) and increased $\mathrm{K}_{\mathrm{rel}}$ (solid symbols) conditions. Solid line was produced by curve fitting of $\mathrm{Ca}_{\mathrm{sys}}=\mathrm{a}+\mathrm{b}(\mathrm{SR} \mathrm{Ca})^{\mathrm{n}}$, where $n=3.5$ for control and rises to 15.0 for increased $K_{\text {rel }}$.

\section{Results}

Inhibition of $S R$ Ca release mechanism. In this part of study, $\mathrm{Ca}^{2+}$ alternans was produced by inhibition of $\mathrm{Ca}$ release, simulating the effects of tetracaine as seen in experiments [7]. In simulations, inhibition of $\mathrm{Ca}$ release was modelled by increasing the subspace $\left[\mathrm{Ca}^{2+}\right]$ concentration required for $\mathrm{Ca}^{2+}$ release from the SR (a parameter called $\mathrm{K}_{\mathrm{rel}}$ in the model) by $20 \%$. The results are shown in Figure 1. Figure 1A is the space-time plot of the colour-scaled $\mathrm{Ca}^{2+}$ concentration of the $25 \mathrm{RyR}$ elements of the cell, in response to a series of stimuli with standard (the first two stimuli) and increased $\mathrm{K}_{\text {rel }}$ (the following stimuli). With standard $\mathrm{K}_{\mathrm{rel}}$, each stimulus evokes a SR release producing a $\mathrm{Ca}$ wave across the cell. However, when $\mathrm{K}_{\mathrm{rel}}$ is increased, $\mathrm{Ca}$ alternans is produced as every two stimuli can produce one Ca wave across the cell. This can be seen more clearly in Figure $1 \mathrm{~B}$, which plots the time traces of cytoplasmic $\mathrm{Ca}$
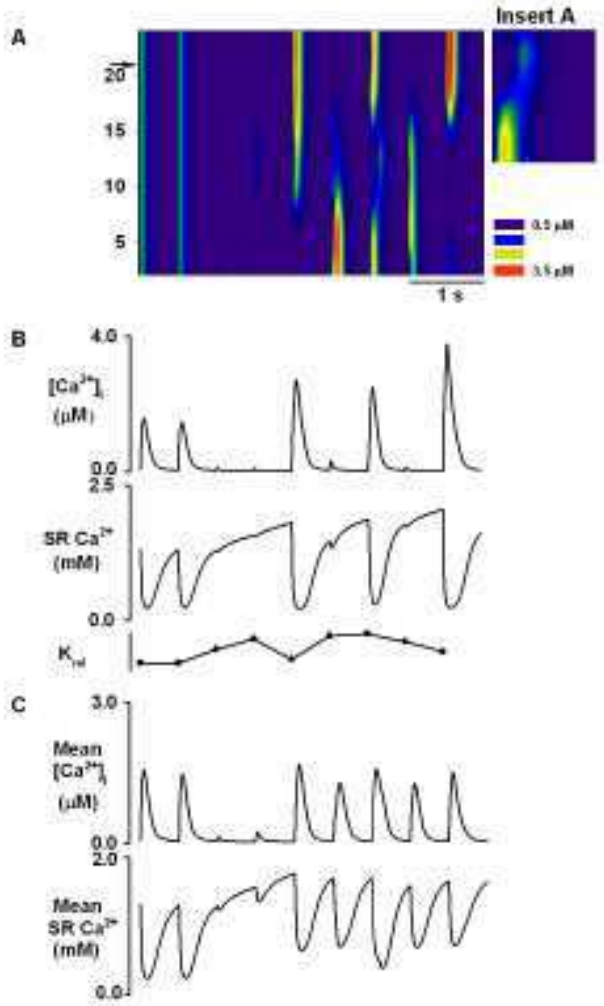

Figure 2. Simulated $\mathrm{Ca}^{2+}$ alternans by increasing $\mathrm{K}_{\text {rel }}$ nonuniformly across the cell. $\mathrm{Ca}^{2+}$ transients were produced by a sequence of $1 \mathrm{~Hz} 100 \mathrm{~ms}$ depolarising pulses from a holding potential of $-40 \mathrm{mV}$ to $0 \mathrm{mV}$ (indicated above the linescan). $\mathrm{K}_{\text {rel }}$ was increased in a random pattern by an average of $20 \%$ across the cell after the first 2 pulses. The pattern in which $\mathrm{K}_{\text {rel }}$ was raised also varied at random from stimulus to stimulus. A) Space-time plot of colourscaled cytoplasmic $\mathrm{Ca}^{2+}$ concentration across the cell. After increase of $\mathrm{K}_{\text {rel }}$, localised and fragmented $\mathrm{Ca}^{2+}$ alternans developed after a short period of $\mathrm{Ca}^{2+}$ release inhibition. Insert A: propagation of local $\mathrm{Ca}^{2+}$ wave. B) Top panel: Traces of cytoplasmic $\mathrm{Ca}^{2+}$ recorded from one element marked by the arrow. Middle panel: associated trace of $\mathrm{SR} \mathrm{Ca}^{2+}$ from the same element. Bottom panel: trace of $\mathrm{K}_{\text {rel }}$. C) Top panel: Trace of mean cytoplasmic $\mathrm{Ca}^{2+}$ averaged across the cell. Bottom panel: Time trace of SR $\mathrm{Ca}^{2+}$ averaged across the cell.

transient recorded from a RyR element. It is shown that after 2 control stimuli, the amplitude of $\mathrm{Ca}$ transient is reduced after increasing $\mathrm{K}_{\text {rel }}$. Then there follows clear alternans of $\mathrm{Ca}^{2+}$ release, which produces one large $\mathrm{Ca}$ transient followed by a small $\mathrm{Ca}$ transient. 


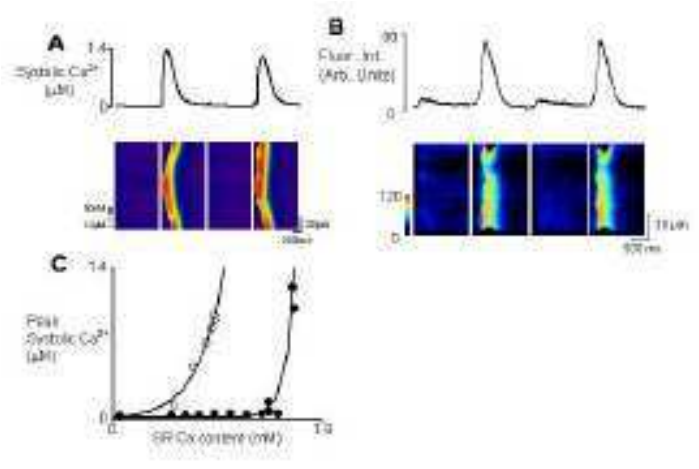

Figure 3. Model generated $\mathrm{Ca}^{2+}$ alternans by small depolarising pulses. The model is stimulated by a series of $1 \mathrm{~Hz} 100 \mathrm{~ms}$ pulses from a holding potential of $-40 \mathrm{mV}$ to $-20 \mathrm{mV}$. L-type calcium current $\left(\mathrm{i}_{\mathrm{CaL}}\right.$ ) was also randomly blocked by $90 \%$ for 17 out of 25 elements which vary from stimulus to stimulus. A) Top panel: time trace of cytoplasmic $\mathrm{Ca}^{2+}$ averaged across model of cell. Lower panel: space-time plot of colour-scaled cytoplasmic $\mathrm{Ca}^{2+}$ concentration across cell that correspond to the transients marked $\mathrm{a}$ and $\mathrm{b}$ above. $\mathrm{B}$ ) Top panel: experimental recording of Fluo 3 fluorescence averaged across a cardiac cell in response to a series of small stimulus pulses as described in (A). Bottom panel: line scan images of Fluo 3 fluorescence across a rat ventricular cell corresponding to transients above. C) Relationship between SR $\mathrm{Ca}^{2+}$ content and systolic $\mathrm{Ca}^{2+}$ in the cytoplasmic space for control (open symbols) and small pulse (solid symbols) conditions in the model. Solid line was produced by curve fitting of Casys $=a+b(S R C a)^{n}$. $n=3.5$ for control and rises to 18.4 for small pulse depolarisation.

Corresponding to the $\mathrm{Ca}$ transient alternans is the fluctuation of SR Ca content as shown in the bottom panel of Figure 2B. In the figure, it is shown that each stimulus depletes the SR, followed by a refilling period. After raising $\mathrm{K}_{\mathrm{rel}}$, the period with very small transients allows the SR to fill to the point where alternans can occur. Before small releases SR content is less than before large releases. In order to underpin the mechanisms underlying the genesis of cytoplasmic $\mathrm{Ca}$ transient alternans, we measured the relationship between the SR Ca content and the amplitude of cytoplasmic $\mathrm{Ca}$ transient. The results are shown in Figure 1C. There are two plots $\mathrm{SR} \mathrm{Ca}^{2+}$ release vs. SR $\mathrm{Ca}^{2+}$ content under control conditions (open symbols) and with $\mathrm{K}_{\text {rel }}$ raised (filled symbols). These plots follow SR filling from the empty state (not shown). It is clear that at high $\mathrm{Ca}^{2+}$ content the steepness is increased when $\mathrm{K}_{\mathrm{rel}}$ is raised (the fitted lines have the formula $\mathrm{Ca}_{\text {syst }}=\mathrm{a}+\mathrm{b}(\mathrm{SR} \mathrm{Ca})^{\mathrm{n}}$, in control $\mathrm{n}=3.5$, rising to 15.0 when $\mathrm{K}_{\text {rel }}$ is raised), this extra gain of $\mathrm{Ca}^{2+}$ release (i.e., the ratio between change in SR content and the resultant change in SR release) allows large changes of released $\mathrm{Ca}^{2+}$ for relatively minor changes of SR content.

However, the Ca release shown in Figure 1A does not present spatial heterogeneity i.e. Ca release is uniform across the cell, which is different to experimental observations [7]. In order to reproduce fragmented $\mathrm{Ca}$ release across the cell, it is necessary to increase $\mathrm{K}_{\mathrm{rel}}$ in a random pattern across the SR release units. On average the increase of $\mathrm{K}_{\mathrm{rel}}$ is the same as in Figure 1 but now it varies among the 25 release units randomly. This random pattern of raising the threshold also changes randomly with each stimulus, reflecting the dynamic variation of combined stochasticity of a cluster unitary L-type and RyR channels in each of the element. The results are shown in Figure 2. Figure 2A shows two control stimuli and very small transients just after $\mathrm{K}_{\mathrm{rel}}$ is raised. However, local alternans then follow as the release profile fragments develop. This is consistent with experimental data [7], large releases are associated with miniwaves of $\mathrm{Ca}^{2+}$ release (see insert). The fragmented $\mathrm{Ca}$ wave can be explained by local variations in $\mathrm{SR} \mathrm{Ca}^{2+}$ content as shown in Figure 2B, which plots the time trace of recorded SA Ca content at the element marked by the arrow in Figure 2A. In Figure 2B, the top panel plots the time trace of recorded cytoplasmic $\mathrm{Ca}$ transient from this element whilst the middle panel shows the associated SR $\mathrm{Ca}$ content. During local alternans SR Ca is relatively high locally before large releases and relatively low before small releases. If these differences are determined by SR Ca content, $\mathrm{K}_{\text {rel }}$ also has a role as shown in the bottom panel. In Figure 2B, the second large release after raising $\mathrm{K}_{\mathrm{rel}}$ arises from a slightly higher SR Ca content than the first large release but is clearly smaller. This is due to a higher level of $\mathrm{K}_{\text {rel }}$ (shown varying at this point in the model cell in the bottom trace of Figure 2B). Thus $\mathrm{SR}$ content and $\mathrm{K}_{\text {rel }}$ combine to determine local $\mathrm{Ca}$ release. Due to regions alternating out of phase, the degree of alternans averaged over the whole cell is small as shown in Figure 2C.

Cytoplasmic $\mathrm{Ca}^{2+}$ transients produced by small depolarisations. In this part of study, we used a small depolarisation pulse to produce $\mathrm{Ca}$ alternans, the same protocol as used experimentally [8]. The simulation data was then used to analyse the underlying mechanisms. The results are shown in Figure 3. In simulations, a series of small stimulus pulses, lead to sustained alternans of cytoplasmic $\mathrm{Ca}^{2+}$ transients (Figure $3 \mathrm{~A}$ ). However, the degree of alternans averaged over the whole cell is much greater than in Figure 2. This is because either the whole cell releases $\mathrm{Ca}^{2+}$ or very little of the cell responds. However, unlike Figure 2, the large release involves $\mathrm{Ca}^{2+}$ 
wave propagation, very similar to the experimental data shown in Figure 2B [8].

The genesis of cytoplasmic $\mathrm{Ca}$ alternans can also be attributed to the stiff relationship between systolic $\mathrm{Ca}^{2+}$ and $\mathrm{SR} \mathrm{Ca}^{2+}$ content as shown in Figure 3C. The fitted lines in the figure have $\mathrm{n}$ set to 3.5 in control and 18.4 during small depolarisations.

The model also reproduces the biphasic release we see experimentally when using small pulses [8]. The trace of systolic $\mathrm{Ca}^{2+}$ in the top panel of Figure 3A shows two successive stimuli with small initial releases. A second phase of release (produced by wave propagation) follows in the first stimulus. This fits well with data from cells under similar experimental conditions [8].

Our simulation data also suggests that alternans produced by small depolarisations also relies on variations of $\mathrm{SR} \mathrm{Ca}$ content and efflux via $\mathrm{Na}^{+}-\mathrm{Ca}^{2+}$ exchange current (NCX) during big and small $\mathrm{Ca}^{2+}$ transients. It is observed that a big $\mathrm{Ca}^{2+}$ transient was associated with a large $\mathrm{SR} \mathrm{Ca}^{2+}$ release (i.e. junctional SR content falls more) and more $\mathrm{Ca}^{2+}$ removal by $\mathrm{NCX}$; while a small $\mathrm{Ca}^{2+}$ transient was associated with less $\mathrm{Ca}^{2+}$ release and less $\mathrm{Ca}^{2+}$ removal by $\mathrm{NCX}$. Before the small release $\mathrm{SR} \mathrm{Ca}^{2+}$ is lower than before the large release. During the time course, there is no change in the amplitude of L-type calcium current implying that the trigger of $\mathrm{Ca}^{2+}$ release is unchanged between large and small releases, and changes in $\mathrm{SR} \mathrm{Ca}^{2+}$ content and $\mathrm{NCX}$ are solely responsible for genesis of $\mathrm{Ca}$ alternans. This, too, is consistent with experimental observations [8].

\section{Discussion and conclusions}

In both cases systolic $\mathrm{Ca}^{2+}$ alternans was generated, which was consistent with previous experimental observations. From the modelling data, the relationship between the SR content and $\mathrm{Ca}^{2+}$ transient was analyzed for normal and alternans conditions. Effects of propagating $\mathrm{Ca}^{2+}$ diffusion waves in generating concordant and dis-concordant $\mathrm{Ca}^{2+}$ alternans in the cell was also analyzed. Our study suggested that the intracellular $\mathrm{Ca}^{2+}$ alternans was generated by propagating waves of $\mathrm{Ca}^{2+}$ release and sustained through alternation of $\mathrm{SR} \mathrm{Ca}^{2+}$ content that has a stiff relationship with the $\mathrm{Ca}^{2+}$ transient. And such mechanisms underlying the genesis of $\mathrm{Ca}$ alternans are model independent as similar behaviours have been observed in a model with a much finer spatial resolution [9]. To conclude, this study provides novel and fundamental insights to understand mechanisms that may underlie intracellular $\mathrm{Ca}^{2+}$ alternans without the need for refractoriness of L-type Ca or RyR channels under rapid pacing.

\section{Acknowledgements}

The work was supported by the BBSRC (UK, BBS/B/1678X), NSFC (China, 60571025) and 863 Program (China, 2006AA01Z308).

\section{References}

[1] Kodama M, Kato K, Hirono S, Okura Y, Hanawa H, Yoshida T, Hayashi M, Tachikawa H, Kashimura T, Watanbe K, and Aizawa Y. Linkage Between Mechanical and Electrical Alternans in Patients with Chronic Heart Failure. J Cardiovasc Electrophysiol 2004; 15:295-299.

[2] Armoundas AA, Tomaselli GF, Esperer HD. Pathophysiological basis and clinical application of Twave alternans. J Am Coll Cardiol 2002; 40:207-217.

[3] Eisner DA, Isenberg G, Sipido KR. Normal and pathological excitation-contraction coupling in the heart -- an overview. J Physiol 2003; 546:3-4.

[4] Pogwizd SM, Hoyt RH, Saffitz JE, Corr PB, Cox JL, Cain ME. Reentrant and focal mechanisms underlying ventricular tachycardia in the human heart. Circulation 1992; 86:1872-1887.

[5] Sipido KR, Volders PGA, de Groot SHM, Verdonck F, van de Werf F, Wellens HJJ, Vos MA. Enhanced $\mathrm{Ca}^{2+}$ release and $\mathrm{Na} / \mathrm{Ca}$ exchange activity in hypertrophied canine ventricular myocytes: a potential link between contractile adaptation and arrhythmogenesis. Circulation 2000; 102:2137-2144.

[6] Dumitrescu C, Narayan P, Efimov IR, Cheng Y, Radin MJ, McCune SA, Altschuld RA. Mechanical alternans and restitution in failing SHHF rat left ventricles. Am J Physiol 2002; 282:H1320-H1326

[7] Díaz ME, O'Neill SC, Eisner DA. Sarcoplasmic reticulum calcium content fluctuation is the key to cardiac alternans. Circ Res 2004; 94:650-656.

[8] Díaz, ME, Trafford AW, O'Neill SC, Eisner DA. Measurement of sarcoplasmic reticulum $\mathrm{Ca}^{2+}$ content and sarcolemmal $\mathrm{Ca}^{2+}$ fluxes in isolated rat ventricular myocytes during spontaneous $\mathrm{Ca}^{2+}$ release. J Physiol (Lond) 1997; 501:3-16.

[9] Tao T, O'Neill SC, Diaz ME, Li YT, Eisner DA, Zhang H. Alternans od cardiac calcium cycling in a cluster of ryanodine receptors: a simulation study. Am J Physiol 2008; 295: H598-H609.

Address for correspondence

Henggui Zhang

Biological Physics Group, School of Physics \& Astronomy

The University of Manchester

Manchester, M13 9PL

UK

E-mail: hemggui.zhang@manchester.ac.uk 